\title{
Ensayos preliminares para determinar la fijación biológica de nitrógeno de Adesmia bicolor a través del método de la abundancia natural
}

\author{
Preliminary tests to determine the biological fixation of nitrogen in Adesmia bicolor \\ through the method of natural abundance
}

Mercedes, Panzitta; Rosana, Malpassi; Sara, Basconsuelo; Verónica, Pérez; Juan, Gorjon; Damaris, Fitzimons, Luciana, Bianco*

\begin{abstract}
RESUMEN
En regiones semiáridas de Argentina se está evaluando Adesmia bicolor (Leguminosae) como especie forrajera de ciclo indefinido, ya que es una leguminosa herbácea perenne de hábito rastrero y con elevada capacidad para fijar nitrógeno. Debido a la falta de información respecto a la capacidad que posee para fijar nitrógeno atmosférico en su ecosistema natural, se propuso como objetivos de trabajo: I) Cuantificar la biomasa aérea y el contenido de nitrógeno producidos por A. bicolor durante su ciclo de vida, II) Cuantificar la biomasa aérea y el contenido de nitrógeno producidos por las gramíneas seleccionadas para utilizar como especies de referencia: Sorghum halepense, Paspalum notatum y Cynodon dactylon durante su ciclo de vida y III) Determinar si estas especies son apropiadas para ser utilizadas en la cuantificación del nitrógeno fijado biológicamente por A. bicolor con el método de la abundancia natural. En Villa Rumipal (provincia de Córdoba, Argentina) se recolectaron muestras de A. bicolor junto con tres especies gramíneas: $P$. notatum, $S$. halepense y $C$. dactylon en las etapas vegetativa, floración y fructificación. Las gramíneas seleccionadas como referencia presentan características fenológicas y ciclos de vida similares a la especie leguminosa en estudio. Se evaluó biomasa aérea y contenido de nitrógeno a través del método de Kjeldahl. Si bien en este estudio no se determina la eficiencia de la fijación biológica de nitrógeno en A. bicolor, se puede predecir (a partir de los datos de biomasa aérea y contenido de nitrógeno) que esta se vería disminuida después de la floración. En las especies utilizadas como referencia se observa un notable incremento de biomasa a lo largo de su ciclo de vida. Luego de analizar los datos obtenidos, se puede concluir que la especie de referencia más apropiada para evaluar el contenido de nitrógeno es $P$. notatum, ya que explora un volumen de suelo similar a $A$. bicolor. S, halepense y $C$. dactylon presentaron un contenido de nitrógeno mayor que la leguminosa estudiada, lo que puede atribuirse a que sus sistemas subterráneos son más vigorosos y, por lo tanto, presentan mayor capacidad para extraer nitrógeno del suelo.

Palabras clave: Adesmia bicolor, gramíneas, especies de referencia, nitrógeno
\end{abstract}

\begin{abstract}
In semi-arid regions of Argentina, Adesmia bicolor (Leguminosae) is being evaluated as an indefinite cycle forage species, as it is a perennial herbaceous legume with a potentially productive stoloniferous habit and high capacity to fix nitrogen. Due to the lack of information regarding the capacity it has to fix atmospheric nitrogen in its natural ecosystem, the following objectives were proposed: I) To quantify aerial biomass and nitrogen content produced by A. bicolor during its life cycle, II) To quantify aerial biomass and nitrogen content produced by selected grasses to be used as reference species: Sorghum halepense, Paspalum notatum and Cynodon dactylon during their life cycle and III) To determine if these species are appropriate to be used in the quantification of nitrogen fixed biologically by A. bicolor with the method of natural abundance. In Villa Rumipal (province of Córdoba, Argentina) samples of $\mathrm{A}$. bicolor were collected together with three grass species: P. notatum, $\mathrm{S}$. halepense, and $\mathrm{C}$. dactylon in the vegetative, flowering and fruiting stages. The grasses selected as reference species show phenological characteristics and life cycles similar to the legume specie under study. Aerial biomass and nitrogen content were evaluated through Kjeldahl method. Although the efficiency of biological nitrogen fixation in A. bicolor is not determined in this study, it can be predicted (based on aerial biomass data and nitrogen content) that it would be reduced after flowering stage. In the species used as a reference there is a notable increase in biomass throughout its life cycles. After the data collected was analyzed, it can be concludes that the most appropriate reference species to evaluate the nitrogen content is $\mathrm{P}$. notatum, since it explores a volume of soil similar to $\mathrm{A}$. bicolor. $\mathrm{S}$. halepense and $\mathrm{C}$. dactylon had a higher nitrogen content, this can be attributed to the fact that their underground systems are more vigorous and, therefore, are more able to extract nitrogen from the soil.
\end{abstract}

Key words: Adesmia bicolor, grasses, reference species, nitrogen.

1 Laboratorio de Morfología Vegetal, Facultad de Agronomía y Veterinaria, Universidad Nacional de Río Cuarto, Ruta Nac. 36 - km 601. 5800, Río Cuarto, Provincia de Córdoba, Argentina.

Autor corresponsal: 1bianco@ayv.unrc.edu.ar.

Fecha de Recepción: 11 diciembre, 2018.

Fecha de Aceptación: 02 de febrero 2019.

DOI: http://dx.doi.org/10.4067/S0718-34292018005002301. Publicado en línea: 12-marzo-2019. 


\section{Introducción}

Las leguminosas nativas son componentes importantes de nuestros ecosistemas áridos y semiáridos (Ardley, 2017). En suelos pobres de nitrógeno (N) son capaces de asociarse con los rizobios, dando origen a estructuras especializadas denominadas nódulos, donde se produce la fijación biológica de nitrógeno (FBN) (Ampomah et al., 2017; Ardley, 2017; Sprent et al., 2017; Afkhami et al., 2018). La evaluación precisa del N fijado simbióticamente por leguminosas nativas es importante en varios aspectos, entre ellos, la función de estas especies en el mejoramiento de agro-ecosistemas y en la estructura del suelo, la evaluación de la eficiencia y eficacia de los rizobios que nodulan la leguminosa y el control de plagas y/o enfermedades (Peoples et al., 1989). El principal problema para cuantificar el $\mathrm{N}$ fijado biológicamente es la selección del método apropiado. El método de la abundancia natural de ${ }^{15} \mathrm{~N}$ es uno de los más recomendables y utilizados actualmente (Valles de la Mora et al., 2003). Este método permite evaluar la fijación de $\mathrm{N}$ utilizando dos especies (una leguminosa fijadora de $\mathrm{N}$ y una especie de referencia no fijadora) que coexisten en un mismo lugar sin necesidad de un experimento diseñado previamente (Unkonvich et al., 2008). Las especies que usualmente se utilizan como referencia son gramíneas (Poáceas), leguminosas no nodulantes o sin inocular, cultivos no leguminosos, especies arbóreas y hierbas de hoja ancha (Unkovich et al., 2008).

Adesmia bicolor es una leguminosa nativa perenne, herbácea, de ciclo indefinido, ampliamente distribuida y promisoria por sus características forrajeras (Bianco, 2002; Scheffer-Basso et al., 2002; Veneciano et al., 2005; Vileta et al., 2010; Bianco et al., 2013). Se distribuye en Argentina, sur de Brasil, Chile y Uruguay (Burkart, 1952; Veneciano et al., 2005). En Argentina crece en provincias como Misiones, Corrientes, Entre Ríos, Santa Fe, Tucumán, Río Negro, Córdoba y San Luis, específicamente en ambientes serranos y pedemontanos (Bianco, 2002). Es capaz de crecer en suelos arenosos hasta gravillosos, incluso arcillosos, con gran habilidad colonizadora de suelos degradados o subsuelos expuestos por erosión o remoción mecánica (INIA, 1992). Nuestro equipo de trabajo está investigando la FBN en A. bicolor desde el año 2006. Hasta el momento, se ha estudiado la eficiencia de FBN en condiciones de invernadero, la diversidad de rizobios capaces de nodular sus raíces, la presencia de estos en el suelo de la región central de Argentina y la capacidad que presentan distintas poblaciones de esta especie para fijar biológicamente $\mathrm{N}$ atmosférico. Se ha determinado que $A$. bicolor es nodulada por bacterias que pertenecen al género Rhizobium, el mecanismo de infección es crack entry y los nódulos son tipo aeschynomenoide (Vileta $e t$ al., 2010; Bianco et al., 2013). Debido a la falta de información respecto a la capacidad que posee para fijar $\mathrm{N}$ atmosférico en su ecosistema natural a lo largo de su ciclo de vida, se propuso como objetivos de trabajo: I) Cuantificar la biomasa aérea y el contenido de nitrógeno producidos por A. bicolor durante su ciclo de vida, II) Cuantificar la biomasa aérea y el contenido de nitrógeno producidos por las gramíneas seleccionadas para utilizar como especies de referencia: Sorghum halepense, Paspalum notatum y Cynodon dactylon durante su ciclo de vida, y III) Determinar si estas especies son apropiadas para ser utilizadas en la cuantificación del nitrógeno fijado biológicamente por $A$. bicolor con el método de la abundancia natural.

El área de estudio comprende el centro-sur de Córdoba, que se caracteriza por presentar clima templado, suelos bien drenados, profundos, aptos para cultivos, pasturas y campos naturales de pastoreo. Las precipitaciones declinan desde los $900 \mathrm{~mm}$ en el este hasta $200 \mathrm{~mm}$ al oeste, dando lugar de este modo a un régimen subhúmedo en el sector oriental y un régimen árido en el sector occidental. Las mayores precipitaciones ocurren en el semestre estival (octubre-marzo), con picos en enero y diciembre, siendo normalmente los meses más secos junio y julio (Bianco, 2002). El muestreo se realizó en Villa Rumipal (provincia de Córdoba, Argentina) (32 $11^{\circ}$ S y $64^{\circ} 28^{\prime}$ O). Se tomaron muestras de una población de Adesmia bicolor (Poir.) DC y de las especies de gramíneas que fueron utilizadas como referencia: Cynodon dactylon (L.) Pers., Sorghum halepense (L.) Pers y Paspalum notatum Flüggé. Estas han sido seleccionadas por presentar características fenológicas y ciclos de vida similares a la especie leguminosa en estudio. Para cuantificar la FBN se llevaron a cabo muestreos durante las etapas vegetativa, de floración y de fructificación. En cada uno de ellos, para determinar la biomasa aérea de cada especie, se tomaron tres muestras utilizando un aro de 0,25 $\mathrm{m}^{2}$, las que fueron secadas en estufa a $65^{\circ} \mathrm{C}$ durante 72 horas. Posteriormente, se tomaron $100 \mathrm{~g}$ de cada muestra para analizar contenido de $\mathrm{N}$ total a través del método de Kjeldahl (Bremner y Mulvaney, 1982). Los análisis estadísticos fueron realizados con el programa Infostat (Di Rienzo et al., 2018). 
En la Tabla 1 se puede observar que tanto en la etapa vegetativa como en la de floración, la biomasa aérea presenta diferencias estadísticamente significativas ( $\mathrm{p}=0,0447$ y $\mathrm{p}=0,0270$, respectivamente), siendo $S$. halepense diferente con respecto a $A$. bicolor y $P$. notatum, mientras que en la etapa de fructificación $S$. halepense muestra diferencias estadísticamente significativas en comparación con el resto de las especies $(\mathrm{p}=0,0001)$.

En la etapa vegetativa y de floración $A$. bicolor muestra un incremento de biomasa aérea que disminuye en la etapa de fructificación. Esto puede deberse a que la FBN promueve cambios morfo-fisiológicos en las plantas que resultan en la disminución del crecimiento. Los nódulos representan un drenaje adicional de fotoasimilados y nutrientes, compitiendo con otros órganos de la planta (Cassman et al., 1980). Estos resultados concuerdan con estudios realizados en especies del mismo género como $A$. latifolia y A. araujoi (Scheffer-Basso et al., 2000, 2001). Por otro lado, la biomasa aérea producida por A. bicolor puede compararse con la producida por especies de leguminosas del género Trifolium, principalmente Trifolium pratense (Nesheim y Øyen 1994; Huss-Danell et al. 2007). La principal razón de estos resultados pueden ser las semejanzas entre estas leguminosas en la forma de crecimiento (porte bajo y rastrero), la perennidad, mayor crecimiento vegetativo invierno-primaveral y floración primaveral hasta otoñal y su potencial forrajero (Veneciano $e t$ al., 2005)
El contenido de $\mathrm{N}$ en biomasa aérea de las especies estudiadas en la etapa vegetativa (Tabla 1) muestra diferencias estadísticamente significativas entre $S$. halepense, $C$. dactylon y A. bicolor con respecto a $P$. notatum. $(\mathrm{p}=0,0271)$.

En la etapa de floración, el contenido de $\mathrm{N}$ presenta un incremento con respecto al muestreo anterior en todas las especies. Se observan diferencias estadísticamente significativas ( $\mathrm{p}=0,0006)$, siendo $S$. halepense y $C$. dactylon diferentes con respecto al resto de las especies. Por otro lado, en la etapa de fructificación se observa que el contenido de $\mathrm{N}$ en las especies estudiadas disminuye, excepto en $P$. notatum que incrementa considerablemente su valor. Los valores de $\mathrm{N}$ obtenidos presentan diferencias estadísticamente significativas $(\mathrm{p}=0,0098)$, siendo $S$. halepense diferente con respecto a A. bicolor y P. notatum.

Bianco (2013) realiza un ensayo en invernadero para estudiar el patrón de nodulación y la FBN de A. bicolor, en el que observa que la especie es efectiva para fijar $\mathrm{N}$ y que la cantidad que fija se va incrementando a medida que la planta va creciendo. Burkart (1952) cita las especies de Adesmia como inefectivas para fijar N. Sin embargo, Scheffer-Basso et al. (2001) observan una elevada producción de biomasa, nodulación y alta eficiencia para fijar $\mathrm{N}$ en especies de este género. Vileta et al. (2010) estiman que $A$. bicolor presenta una eficiencia de FBN del $60 \%$ en condiciones ideales de invernadero. Estos valores son relativamente altos en comparación con otras leguminosas como Medicago sativa (alfalfa) que

Tabla 1. Biomasa aérea $\left(\mathrm{kg} \mathrm{ha}^{-1}\right)$ y contenido de $\mathrm{N}\left(\mathrm{kg} \mathrm{ha}^{-1}\right)$ de Adesmia bicolor, Paspalum notatum, Sorghum halepense y Cynodon dactylon \pm el error estándar, obtenido en la etapa vegetativa, floración y fructificación. Las diferentes letras indican diferencias estadísticamente significativas entre las especies según análisis de LSD-Fisher con Infostat 2005/P.1 $(\alpha=0,05)$.

\begin{tabular}{|c|c|c|c|}
\hline Etapas de crecimiento & Especie & Biomasa aérea (kg ha-1) & Contenido de $\mathbf{N}\left(\mathrm{kg} \mathrm{ha}^{-1}\right)$ \\
\hline \multirow{4}{*}{ Vegetativa } & Adesmia bicolor & $1290,99 \pm 413,2 a$ & $124,70 \pm 35,78 b$ \\
\hline & Paspalum notatum & $831,60 \pm 113,1 \quad a$ & $10,74 \pm 2,66 \mathrm{a}$ \\
\hline & Sorghum halepense & $4286,63 \pm 2124,20 b$ & $140,69 \pm 84,16 b$ \\
\hline & Cynodon dactylon & $2833,91 \pm 1496,4 \quad a b$ & $164,70 \pm 48,00 \mathrm{~b}$ \\
\hline \multirow{4}{*}{ Floración } & Adesmia bicolor & $1547,1 \pm 218,7 \mathrm{a}$ & $179,46 \pm 11,38 \mathrm{a}$ \\
\hline & Paspalum notatum & $864,6 \pm 332,1$ a & $57,45 \pm 27,79 a$ \\
\hline & Sorghum halepense & $6613,7 \pm 3945,9 b$ & $673,24 \pm 202,84 \mathrm{c}$ \\
\hline & Cynodon dactylon & $4220,65 \pm 6353,73 a b$ & $430,57 \pm 84,67 b$ \\
\hline \multirow{4}{*}{ Fructificación } & Adesmia bicolor & $1502,3 \pm 205,08 \mathrm{a}$ & $94,51 \pm 18,96 \mathrm{a}$ \\
\hline & Paspalum notatum & $1591,6 \pm 706,7$ a & $129,99 \pm 74,27 \mathrm{a}$ \\
\hline & Sorghum halepense & $8122,4 \pm 1859,2 \mathrm{c}$ & $578,11 \pm 266,18 b$ \\
\hline & Cynodon dactylon & $687,05 \pm 247,02 a b$ & $328,26 \pm 26,25 \mathrm{ab}$ \\
\hline
\end{tabular}


fija hasta el $61 \%$ cuando crece en suelos del centro de Argentina (Perticari, 2006). Si bien en este estudio no se determina la eficiencia de la FBN, se puede predecir (a partir de los datos de biomasa aérea y contenido de N) que esta se vería disminuida después de la floración. Esto puede deberse a que las condiciones de crecimiento en el campo no son óptimas como ocurre en el invernadero.

Otro aspecto de interés es la elección de las gramíneas de referencia. Según los datos analizados en este trabajo, se puede concluir que $S$. halepense y $C$. dactylon no constituyen especies de referencia apropiadas para A. bicolor. Peoples et al. (1989) establecen que para la elección de la especie de referencia la suposición principal es que tanto la leguminosa como la especie de referencia deben contener la misma cantidad de $\mathrm{N}$ derivado del suelo en sus brotes. Para que esto sea válido, los dos tipos de plantas deben explorar el mismo volumen de suelo y tener la misma capacidad para extraer y acumular $\mathrm{N}$ en el mismo período de tiempo. En este estudio se observa que el contenido de $\mathrm{N}$ es mayor en $S$. halepense y $C$. dactylon, lo que puede atribuirse a que sus sistemas subterráneos son más vigorosos que el de A. bicolor, con mayor capacidad para extraer $\mathrm{N}$ del suelo.

\section{Perspectivas futuras}

Teniendo en cuenta los resultados de este estudio, sería interesante determinar la cantidad de $\mathrm{N}$ atmosférico que fija $A$. bicolor en simbiosis con los rizobios nativos del suelo mediante el método de la abundancia natural de ${ }^{15} \mathrm{~N}$, utilizando como especie de referencia $P$. notatum.

\section{Literatura citada}

Afkhami, M.E.; Mahler, D.L.; Burns, J.H.; Weber, M.G.; Wojciechowski, M.F.; Sprent, J.; Strauss, S.Y.

2018. Symbioses with nitrogen-fixing bacteria: nodulation and phylogenetic data across legume genera. Ecology, 99: 502.

Ampomah, O.Y.; Mousavi, S.A.; Lindström, K.; Huss-Danell, K. 2017. Diverse Mesorhizobium bacteria nodulate native Astragalus and Oxytropis in arctic and subarctic areas in Eurasia. Systematic and Applied Microbiology, 40: 51-58.

Ardley, J.

2017. Legumes of the Thar desert and their nitrogen fixing Ensifer symbionts. Plant Soil, 410:517-520.

Bianco, C.A.

2002. Growth forms, taxonomy, distribution and uses of Adesmia species (Leguminosae) in Central Argentina. Cramer . Sttutgart, Alemania. 156 p.

Bianco, L.; Angelini, J.; Fabra, A.; Malpassi, R.

2013. Diversity and Symbiotic Effectiveness of Indigenous Rhizobia-Nodulating Adesmia bicolor in Soils of Central Argentina. Current Microbiology, 66: 174-184.

Burkart, A.E.

1952. Las leguminosas argentinas, silvestres y cultivadas. $2^{\text {da }}$ ed. Ed. Acme Agency, Buenos Aires, Argentina. 569 p.

Bremner, J.M.; Mulvaney, C.S.

1982. Nitrogen-Total. In: Methods of soil analysis. Part 2. Chemical and microbiological properties. American Society of Agronomy, Inc. and Soil Science Society of America, Inc. $2^{\text {da }}$ edición. Madison, USA. pp. 595-624.

Cassman, K.G.; Whitney, A.S.; Stockinger, K.R.

1980. Root growth and dry matter distribution of soybean as affected by phosphorus stress, nodulation, and nitrogen source. Crop Science, 20: 239-244.
Di Rienzo, J.A., Casanoves, F., Balzarini, M.G., González, L., Tablada, M., Robledo, C.W.

2018. InfoStat versión 2018. Grupo InfoStat, FCA, Universidad Nacional de Córdoba. Argentina. Disponible en: http//www. infostat.com.ar.

Huss-Danell, K.; Chaia, E.; Carlsson, G.

2007. $\mathrm{N}_{2}$ fixation and nitrogen allocation to above and below ground plant parts in red clover-grasslands. Plant and Soil, 299: 215-226.

INIA.

1992. Leguminosas nativas promisorias: trébol polimorfo y babositas. Boletín de Divulgación del Instituto Nacional de Investigaciones Agropecuarias. Montevideo, Uruguay.

Nesheim, L.; Øyen, J.

1994. Fijación de nitrógeno por trébol rojo (Trifolium pratense L.) cultivado en mezclas con Timothy (Phleum pratense L.) a diferentes niveles de fertilización nitrogenada. Acta Agriculturae Scandinavica, Section B, Silo planta Science, 44: 28-34.

Peoples, M.B.; Faizah, A.W.; Rerkasem, B.; Herridge, D.F. 1989. Methods for evaluating nitrogen fixation by nodulated legumes in the field. Monog No 11. Centre for International Agricultural Research (ACIAR), Canberra, Australia. 76 p.

Perticari, A

2006. Pasturas de alfalfa: importancia de una adecuada inoculación. $2^{\text {do }}$ Congreso Nacional de Conservación y Uso de Forrajes. INTA Castelar - Buenos Aires, Argentina. pp $1-5$.

Scheffer-Basso, S.M.; Voss, M.; Ávila Jacques, A.V. 2001. Nodulação e fixação biológica de nitrogênio de Adesmia latifolia e Lotus corniculatus em vasos de Leonard. Revista Brasileira de Zootecnia, 30: 687-693. 
Scheffer-Basso, S.M; Ávila Jacques, A.V.; Dall'Agnol, M. 2002. Aloçcao da biomassa e correlaçes morfofisiologicas em leguminosas forrageiras com hábitos de crescimento contrastantes. Revista de Scientia Agrícola, 59: 629-634.

Sprent, J.I.; Ardley, J.; James, E.K.

2017. Biogeography of nodulated legumes and their nitrogenfixing symbionts. New Phytologist, 215:40-56.

Unkonvich, M.; Herridge, D.; Peoples, M.; Cadisch, G.; Boddey,

B.; Giller, K.E.; Alves, B.; Chalk, P.

2008. Measuring plant associated nitrogen fixation in agricultural systems. Ed. Center for Agricultural Research, Canberra, Australia. 258 p.
Valles de la Mora, B.; Cadisch, G.; Schunemann, A.A. 2003. Comparación de metodologías de isótopos para evaluar fijación de nitrógeno atmosférico y su destino en suelos y plantas. Agrociencia, 37: 117-128.

Vileta, D.; Grosso, M.; Fondevila, M.

2013. Nutritive value for ruminants of two herbaceous South-American native legumes: Adesmia bicolor and A. macrostachya. Animal Production Science, 54: 125-130.

Veneciano, J.H.; Frasinelli, C.A..; Kraus, T.A.; Bianco, C.A. 2005. Domesticación de especies forrajeras. $1^{\mathrm{ra}} \mathrm{ed}$. Universidad Nacional de Río Cuarto, Córdoba, Argentina. $64 \mathrm{p}$. 
